\title{
The Cherenkov Telescope Array Project - current status and science goals
}

\section{B. Rudak*}

Centrum Astronomiczne im. Mikołaja Kopernika PAN, Bartycka 18, 00-715 Warszawa, Poland

E-mail: bronekencac.torun.pl

\section{for the CTA Consortium}

The Cherenkov Telescope Array (CTA) Project is a worldwide project aiming at building a large ground-based gamma-ray observatory distributed on two sites located in the northern and southern hemispheres. Its performance will surpass the capabilities of the three Imaging Atmospheric Cherenkov Telescope (IACT) observatories currently in operation: H.E.S.S., MAGIC and VERITAS. The science cases for CTA cover a wide range of topics, from galactic and extragalactic astrophysics to fundamental physics. The CTA Observatory will be the first true open observatory in the history of Very High Energy (VHE) gamma-ray astronomy. It will offer guest observer programme and free access to its data archive.

XI Multifrequency Behaviour of High Energy Cosmic Sources Workshop -MULTIF15-,

25-30 May 2015

Palermo, Italy

${ }^{*}$ BR acknowledges financial support by the National Science Centre grant DEC- 2011/02/A/ST9/00256 to attend MULTIF2015. 


\section{Introduction}

The CTA project ${ }^{1}$ is an initiative to build the next generation ground-based very high energy gamma-ray observatory. The sensitivity of CTA will be better by a factor of up to 10 over the whole energy range covered by H.E.S.S., MAGIC and VERITAS. Moreover, the accessible energy range will be extended, and the angular and energy resolutions will be significantly improved [1]. Very High Energy astronomy will gain a powerful instrument to study the gamma-ray sky. CTA is expected to discover around 1000 sources - a substantial progress with respect to 163 VHE sources known in 2015 according to $\mathrm{TeVCat}^{2}$. The project is realized by an international collaboration - the CTA Consortium, consisting of 32 member countries, with 1270 participants (scientists, engineers and students) from 192 institutes.

The year 2015 marks an important step in the CTA project timeline. Firstly, decisions about site locations of CTA in the south and the north were made and negotiations are now under way with Chile and Spain. Secondly, Key Science Projects (KSPs) and related programmes had been formulated and presented along with detailed technical requirements for external reviews.

\section{Science drivers and requirements}

Three basic science drivers of the CTA project are:

1) Origin of Cosmic Rays. It includes studies of particle accelerators in SNR, PWN, pulsars and micro-QSOs as well as related phenomena - massive SFR and starburst galaxies.

2) Black hole particle accelerators. It includes studies of blazars, radio-galaxies, AGN, GRB as well as of extragalactic background light (EBL), galaxy clusters and cosmic magnetism.

3) Fundamental Physics. It includes search for annihilation signatures of Weakly Interacting Massive Particles (WIMPs) as dark matter particles (DM), search for axion-like particles and testing Lorentz Invariance Violation (LIV).

The following performance capabilities of CTA have been foreseen to address these topics: high sensitivity, with three orders of magnitude of the dynamic range in flux (max,min); wide energy range, from $20 \mathrm{GeV}$ to $100 \mathrm{~s}$ of $\mathrm{TeV}$ (from pulsars, AGN and Cosmology to Pevatrons), see Fig1; energy resolution 10-15\% (spectral lines, shapes of non-thermal continuum at cutoffs); angular resolution, up to $0.02 \mathrm{deg}$, with 10"-20" of source localization (morphology); time resolution superior to Fermi-LAT (flares, GRB, other-short time events), see Fig3; rapid slewing 20 seconds (as above); large field of view, up to $\sim 8$ degree (surveys and serendipitous discoveries of AGN, GRB); surveying capabilities - full-sky survey at $\mathrm{O}(1 \%) \mathrm{Crab}$ in $\sim 1$ year; monitoring capabilities - possible use of sub-arrays for monitoring of AGN.

\section{The CTA Observatory (CTAO) and timeline towards it}

CTAO will be located on two sites, one in each hemisphere, with a full sky coverage at 20-30 $\operatorname{deg} \mathrm{N}, \mathrm{S}$. In the south one site has been selected to start negotiations for an agreement to host CTAO in Armazones (ESO/Chile). In the north similar negotiations will start concerning La Palma (Spain). The southern array will consist of 4 Large Size Telescopes (LSTs, parabolic, $23 \mathrm{~m}$ diameter), 25 Medium Size Telescopes (MSTs, Davies-Cotton design, $12 \mathrm{~m}$ diameter), about 70 Small

\footnotetext{
$1_{\text {www }}$ cta-observatory . org

2 tevcat.uchicago. edu
} 


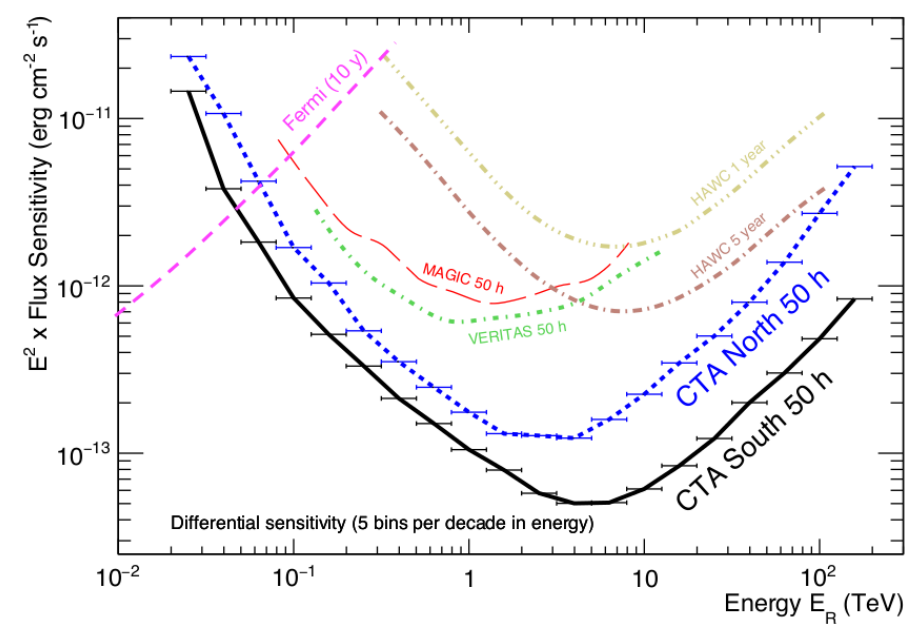

Figure 1: Differential sensitivity (with the criterium of at least 5 sigma C.L. for each of five bins per energy decade) for a point-like source of the CTA candidate arrays in North and South (50 hours of observation) [9]. The sensitivity and the time of observations of other existing gamma-ray observatories are shown for comparison.

Size Telescopes (SSTs, 4 m diameter, both with Davies-Cotton and dual-mirror SchwarzschildCouder optical systems), and probably 25 MSTs with Schwarzschild-Couder system. The array will cover about $10 \mathrm{~km}^{2}$ area. In the north the location has been chosen at Obs. Roque de Muchachos (La Palma, Spain) at $2200 \mathrm{~m}$ asl. It will consist of 4 LSTs and 15 MSTs, covering an area of $\sim 1 \mathrm{~km}^{2}$. LSTs will be sensitive below $\sim 200 \mathrm{GeV}$, reaching a low-energy threshold around 20 $\mathrm{GeV}$, MSTs will cover the core energy domain while SSTs will be sensitive from a few TeV to over $100 \mathrm{TeV}$ (see [12], [3] and [10] for details). Three different sizes ensure that the southern array will be sensitive in a wide energy range. A justification for such preference of the South is that the inner Galaxy and the Galactic Centre belong to the most attractive targets in the VHE sky.

The project started with the Design Phase (between 2008 and 2010) focused on proposals for array layouts, telescope design and science drivers. Then the Preparatory Phase followed (2011 2014) with the development of telescope and component prototypes as well as science performance modeling. In March 2014, CTA Observatory GmbH was created (based on German law) as the legal framework for site negotiations and production phase. Site selection has been completed in 2015 (two sites to cover full sky at $\sim 25-28$ deg N, S). Technical Design Report was submitted in 2015 for critical review by Science and Technical Advisory Committee. In the years 2016 - 2017 pre-production phase will start and the first precursors will be installed. Production phase will then follow from 2018 to 2023.

\section{CTA Science Core Programme}

The core programme for CTAO proposed by the Consortium covers astrophysics, cosmology and some topics of Fundamental Physics. It consists of Dark Matter Programme and 9 Key Science Projects (KSPs): 
- Galactic Centre, - Galactic Plane Survey, - Large Magellanic Cloud Survey,

- Extragalactic Survey, - Transients, - Cosmic Ray PeVatrons,

- Star Forming Systems, - Active Galactic Nuclei, - Clusters of Galaxies.

Three KSPs are devoted to surveys taking advantage of the CTA properties suitable for such projects, as mentioned in Sect.2. In particular, Galactic Plane Survey (GPS) will be a legacy project, expected to discover several hundreds sources, some of them of new types (hence its synergy with Cosmic Ray PeVatrons). Significant increase in the number of PWN and SNR is guaranteed and population studies of both types of sources will gain new quality (MC simulations predict that horizon of detectability in both cases will be $\sim 20 \mathrm{kpc}$ - entire Galaxy). Entire plane will be surveyed to the level of $3.8 \mathrm{mCrab}$, but with inner part studied down to $1.8 \mathrm{mCrab}$ (Fig.2). It will be an ideal

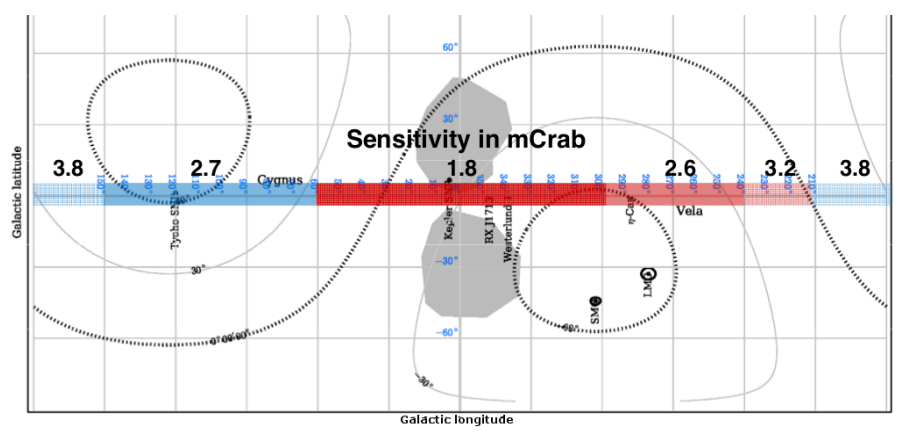

Figure 2: Point-source sensitivities in GPS expected in the ten-year program of the GPS KSP. The surveyed plane will be $\sim 10$ degrees wide (in Gal latitudes) and the survey will be down to the limits given in mCrab unit on top of the GP (various regions are colour coded). The grey shapes depict the Fermi Bubbles.

starting point for specific detailed studies of Galactic sources, like e.g. parts of Galactic Bulge, radio spurs, base of Fermi bubbles and Kepler SNR.

Cosmic Ray PeVatrons KSP should find and unveil the nature of hadronic particle acceleration sites. Ultrarelativistic hadrons (mostly protons) should reach there a total energy per particle of the order of PeV in order to explain the origin of Cosmic Rays below the CR spectral knee. CTA will probe cosmic ray physics in Galactic as well as extragalactic environments.

CTA will be suitable for observing short-timescale events (transients) like GRBs, blazar flarings (see Fig.3). Observations of blazar variability at sub-minute scale will surpass current results [11]. At even shorter time scales - down to several seconds - CTA will probe e.g. VHE emitting regions very close to the jet formation sites in blazars.

\subsection{Dark Matter and Fundamental Physics Programme}

Indirect DM particle searches will be carried out around the Galactic Centre and in dwarf satellites of the Galaxy. CTA will look for signatures of WIMPs as candidate particles for DM mainly as spectral continuum gamma-ray features, and to lesser extent - spectral lines [4]. CTA has good prospects for testing WIMP models with cross section values of the level of thermal relic cross section and mass above $200 \mathrm{GeV}$ (see Fig 4). Moreover, axion-like particle searches are planned, where conversion of gamma-rays to/from axion-like particles can create distinctive features in the spectra of gamma-ray sources and/or increase transparency of the universe by reducing the EBL absorption [5]. Testing of Lorentz invariance violations will also be part of the Programme [6], [7]. 

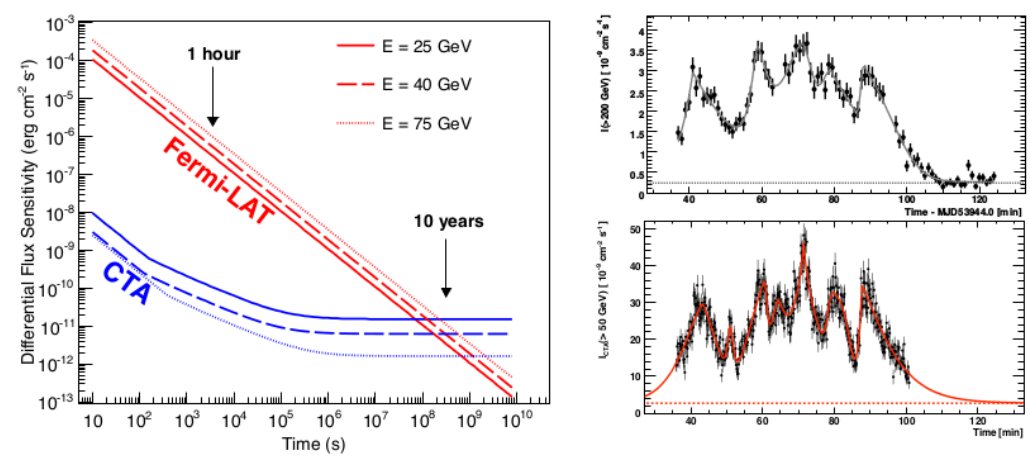

Figure 3: Left: Differential sensitivity of CTA and Fermi-LAT vs. Observation timescale [8]. Right: Top panel - A flare from PKS 2155-304 as detected with the HESS telescopes (July 28, 2006); the data are binned in one minute intervals [2]. Bottom panel - Simulated CTA lightcurve of the 2006 flare, after extension of the red noise to high frequencies was applied (hence new peaks - 2nd and 4th - generated); the data are binned in $7.5 \mathrm{sec}$ intervals [11].

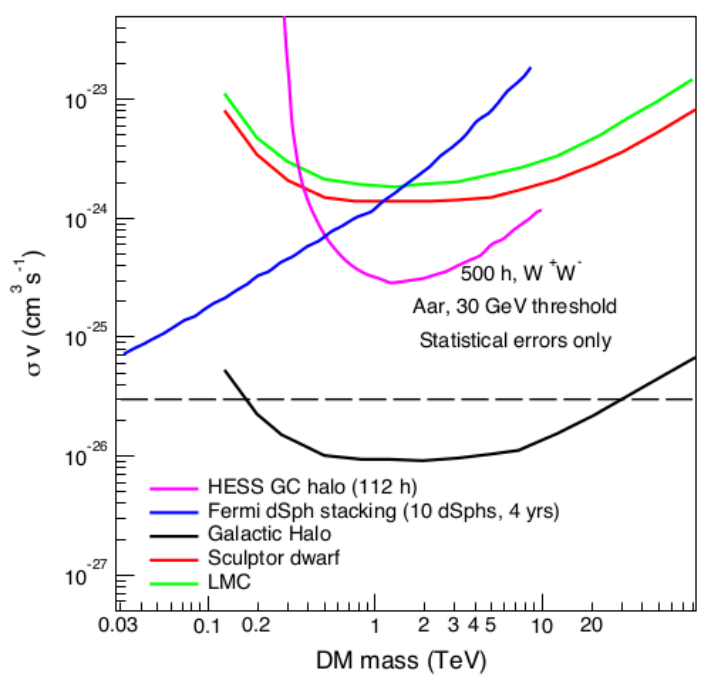

Figure 4: CTA sensitivity to a WIMP annihilation signature as a function of WIMP mass. The dashed horizontal line indicates the likely 'thermal relic' cross-section for a WIMP in the Big Bang universe.

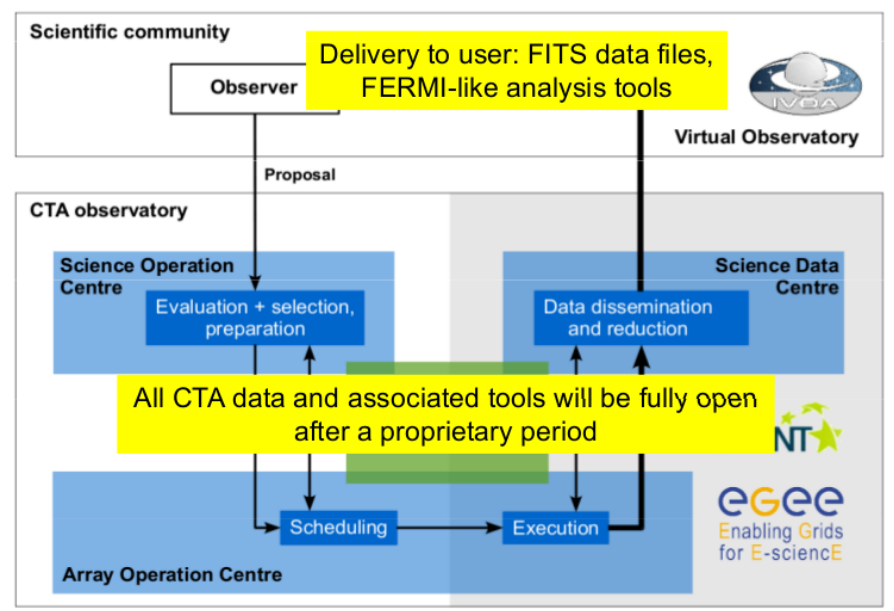

Figure 5: Logical model of CTA data flow. 


\section{KSP and open time scheduling}

CTAO will be the first open observatory in the history of ground-based gamma-ray astronomy. Observation time will be splitted into 'Open time' for scientists of party countries and 'Consortium time' for Key Science Projects. The schematic diagram of how the data flow may look like is shown in Fig.5. All data will become public after a proprietary period (to be determined). Free access to CTAO data archive will be offered.

\section{Acknowledgments}

We gratefully acknowledge support from the agencies and organisations listed under Funding Agencies at this website: http://www.cta-observatory.org/

\section{References}

[1] B.S. Acharya et al., Introducing the CTA concept, APh 2013 (43) 3.

[2] F. Aharonian et al. (H.E.S.S. collaboration), An Exceptional Very High Energy Gamma-Ray Flare of PKS 2155-304, ApJ2007 (664) L71

[3] G. Ambrosi et al. for the CTA Consortium, The Cherenkov Telescope Array Large Size Telescope, The 33rd ICRC 2013, arXiv: $1307.4565 \mathrm{v} 1$

[4] J. Carr et al. for the CTA Consortium, Prospects for Indirect Dark Matter Searches with the Cherenkov Telescope Array (CTA), The 34th ICRC 2015, arXiv: 1508 . $06128 \mathrm{v} 1$

[5] M.A. Sanchez-Conde Cortina et al. for the CTA Consortium, The hunt for axionlike particles with the Cherenkov Telescope Array, White paper contribution for Snowmass 2013 2013, arXiv: 1305.0252

[6] M.K. Daniel et al., Prospects On Testing Lorentz Invariance Violation With The Cherenkov Telescope Array, The 34th ICRC 2015, arXiv: 1508 . $06622 \mathrm{v} 1$

[7] M. Fairbairn et al., The CTA Sensitivity to Lorentz-Violating Effects on the Gamma-Ray Horizon, JCAP2014 (06) 5.

[8] S. Funk, J.A. Hinton and CTA Consortium, Comparison of Fermi-LAT and CTA in the region between 10-100 GeV, APh 2013 (43) 348

[9] T. Hassan et al. for the CTA Consortium, Status of the Medium-Sized Telescope for the Cherenkov Telescope Array, The 34th ICRC 2015, arXiv: 1508 . $06075 \mathrm{v} 1$

[10] T. Montaruli et al. for the CTA Consortium, The small size telescope projects for the Cherenkov Telescope Array, The 34th ICRC 2015, arXiv: 1508 . $06472 \mathrm{v} 1$

[11] H. Sol et al. on behalf of the CTA collaboration, Active Galactic Nuclei under the scrutiny of CTA, APh 2013 (43) 215.

[12] S. Vercellone et al. for the CTA Consortium, The next generation Cherenkov Telescope Array observatory: CTA, NIMPA 2014 (766) 73 